\title{
Using a Multiple Analytical Distribution Filter for Underwater Localization
}

\author{
Dov Kruger ${ }^{a}$, Hongyuan Shi ${ }^{b}$, Yingying Chen ${ }^{c}$, Hongbo Liu $^{c}, \mathrm{Jie}_{\mathrm{Yang}}{ }^{c}, \mathrm{Len}_{\mathrm{Imas}}{ }^{a}$ \\ ${ }^{a}$ Center for Maritime Systems, Stevens Institute of Technology, Hoboken, NJ, U. S. A.; \\ ${ }^{b}$ Center for Decision Technologies, Stevens Institute of Technology, Hoboken, NJ, U. S. A.; \\ ${ }^{c}$ Department of Electrical and Computer Engineering, Stevens Institute of Technology, Hoboken, NJ, U. S. A.
}

\begin{abstract}
This paper presents a high efficiency algorithm, Multiple Analytical Distribution Filter (MADF), to estimate location for underwater navigation. Using small grid sampling around candidate areas of high probability, MADF computes probabilities directly from the known analytical distributions of each beacon. The algorithm is deterministic and achieves similar results to particle filters, but at a lower computational cost in our tests. MADF and particle filters represent improvements over Kalman Filters for environments characterized by non-Gaussian noise distribution.
\end{abstract}

Keywords: Localization, underwater navigation, particle filter, MADF

\section{INTRODUCTION}

Unmanned Underwater Vehicles (UUVs) and autonomous sensors can collect an enormous amount of data underwater. In order to be useful, the data must be correlated to an accurate location, and the higher the precision and density of the data, the more desirable it is to have a high resolution location to match. Localization of UUVs is also important for accurate path planning, determining how close the vehicle is to the desired path and taking corrective action if necessary.

On the surface, GPS makes accurate localization relatively easy. Underwater, however, electromagnetic signals do not penetrate, and UUVs therefore require an alternate navigational system. Methods of navigation include Long Base Line (LBL) acoustic beacons, dead reckoning, digital velocimetry, and inertial navigation systems (INS). LBL beacons provide range estimates by using the speed of sound in water and measuring the time for a signal to travel from vehicle to beacon and back (round trip) or from the beacon to the vehicle (one-way trip). In either case, an accurate assessment of the speed of sound in water is necessary; this depends on the salinity, temperature, and pressure, as well as potentially the vertical profile of sound speed. ${ }^{1}$ Dead reckoning (DR) is a common technique for estimating the distance traveled by multiplying the speed by the time traveled. DR is relatively inaccurate because the exact speed and heading of the UUV are difficult to determine, and because the water itself may be moving. Digital Velocimetry (DV) uses Doppler SONAR to measure motion relative to the bottom, and get a direct measurement of velocity rather than estimating it. Inertial navigation systems use sensors to measure acceleration which is integrated twice to yield position. Some combination of these navigational inputs can be used to determine a position. The question is then how to most efficiently transform navigational data into an estimate of location.

There are a number of algorithms for localization given a sequence of navigational inputs. ${ }^{2,3}$ Kalman filters model each variable as normally distributed, so that a position in a 2D space is an oval, in a 3D an ovoid. For non-Gaussian distributions, Kalman does not preserve all the information. ${ }^{2,4}$ Another classic algorithm, multilateration, computes a least-squares fit of the distance to each beacon without calculating the probabilities. ${ }^{5,6}$ Particle filters offer a way to capture arbitrary distributions by sampling the distribution in multiple positions, estimating the most likely location as the mean of the particles..$^{2,3,7-10}$ In order to propagate forward in time,

Further author information: (Send correspondence to D. Kruger)

D. Kruger: E-mail: dov.kruger@gmail.com

H. Shi: E-mail: hshi@stevens.edu

Y. Chen: E-mail: Yingying.Chen@stevens.edu

Unmanned/Unattended Sensors and Sensor Networks VI, edited by Edward M. Carapezza,

Proc. of SPIE Vol. 7480, 74800T · ( 2009 SPIE · CCC code: 0277-786X/09/\$18 · doi: 10.1117/12.834893

Proc. of SPIE Vol. $748074800 T-1$ 
each particle is randomly transformed according to the physical model of the vehicle's motion. Thus, for example, if the vehicle has a heading of 30 degrees, and a speed of $1 \mathrm{~m} / \mathrm{s}$, the particles are all shifted at each time step, $\Delta t$, by the vector, $\left(\cos 30^{\circ}, \sin 30^{\circ}\right) \Delta t$, with random error added on according to the modeled motion error, which might include course steering error, distance error, and for a vehicle in a fluid, environmental currents and random drift. In order to maintain an accurate coverage of the distribution, particle filters must continually discard the particles that have drifted into unrepresentative low probability areas, and resample with new particles representing high probability regions. A grid approach is appealing since it is deterministic and the resulting maximum error can also be computed in advance based on the grid resolution. However a grid is computationally expensive because it must maintain a large area to guarantee coverage of the high probability region(s).

Based upon our experience with acoustic navigation using LBL beacons, we have proposed a new algorithm, and equivalent to a particle filter but retaining the deterministic behavior of a high resolution grid method. Because the algorithm maintains and intersects multiple individual distributions, each of which can be described analytically, we call it a Multiple Analytical Distribution Filter (MADF). ${ }^{11}$ The initial version of MADF used multiple rectangles to track intersecting regions of distribution fields, which involved a large number of cases. In this paper we present a simpler variant using a small sampling grid around each intersection.

In the following sections, we will briefly describe the method of MADF and construct simulation tests to compare its accuracy and efficiency to particle filters.

\section{ALGORITHMIC APPROACH}

\subsection{Problem Overview for Underwater Navigation}

The LBL acoustic beacons are based on acoustic modems. A moving platform sends a short ping to a set of named beacons and they reply after a duration of fixed length $\tau_{b}$. The total time of travel is thus twice the distance between the beacon and the platform plus the $\tau_{b}$ lag time. The location of the beacons is known based on GPS reading and the anchor equipment when initially deployed. Consider a set of $k$ LBL beacons fixed at locations $P=\left\{P_{i}, 1 \leq i \leq k\right\}$, where $P_{i}=\left\{x_{i}, y_{i}, z_{i}\right\}$ represents the coordinates of beacon $i$. Over a window of $t$ seconds, a set of beacons $B(t)$ can be clearly heard. Their signals $\left\{s_{i}, i \in B(t)\right\}$ are processed and the corresponding distances are $\left\{r_{i}\right\}$. Each signal propagation is assumed to have normally distributed error, with a known standard deviation $\sigma_{r}$ based on signal types and environmental conditions. Thus the estimate from an acoustic beacon can be viewed as an annular ring of probability around the known beacon position,

$$
p(x, y, z)=\frac{1}{\sqrt{2 \pi}} \exp \left\{-\frac{\left(r-r_{i}\right)^{2}}{2 \sigma_{r}^{2}}\right\}, \text { and } r=\sqrt{\left(x-x_{i}\right)^{2}+\left(y-y_{i}\right)^{2}+\left(z-z_{i}\right)^{2}}
$$

where $r_{i}$ is the measured distance from the beacon, and $(x, y, z)$ is the location whose probability is to be computed.

Dead reckoning (DR) is used to combine beacon ranges at different times. There are three errors associated with dead reckoning, in the steering angle, the speed traveled, and a random drift due to environmental currents. The errors can be reduced by continuously measuring accelerations (with an IMU) or the speed relative to the ground (with a DVL). We model a DR estimation of a displacement between points $A$ and $B$ as

$$
\vec{D}_{A B}=(\vec{D}+\delta \vec{D})+(\vec{D}+\delta \vec{D}) \times \delta \vec{\theta}+\vec{\delta}_{e n v} .
$$

The decomposition is illustrated in Fig. 1a. The first term, $\vec{D}+\delta \vec{D}$, represents the distance estimation and the error from speed estimation, $\delta \vec{D}$. The second term, $(\vec{D}+\delta \vec{D}) \times \delta \vec{\theta}$, results from the error of the steering angle

estimation, $\delta \vec{\theta}$. The third term, $\vec{\delta}_{\text {env }}$, models the the random drift due to the environmental currents, which is a function of time and location. In our analysis we only consider the first two terms. Figure $1 \mathrm{~b}$ illustrates a sample case of dead reckoning errors for a 10m DR track (the errors are exaggerated for clarity). We model the DR error as a bivariate normal so it is analytically tractable. The standard deviation for steering, $\sigma_{\text {steer }}$, at time $t$ is approximated as

$$
\sigma_{D R}(t)=\sqrt{\sigma_{D}^{2}(t)+\left(D(t) \cdot \sigma_{\text {head }}\right)^{2}}
$$




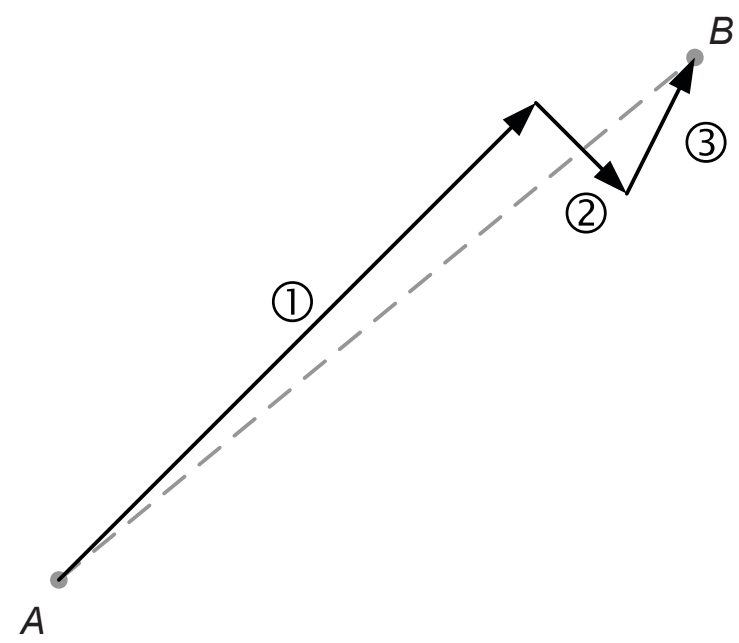

(a)

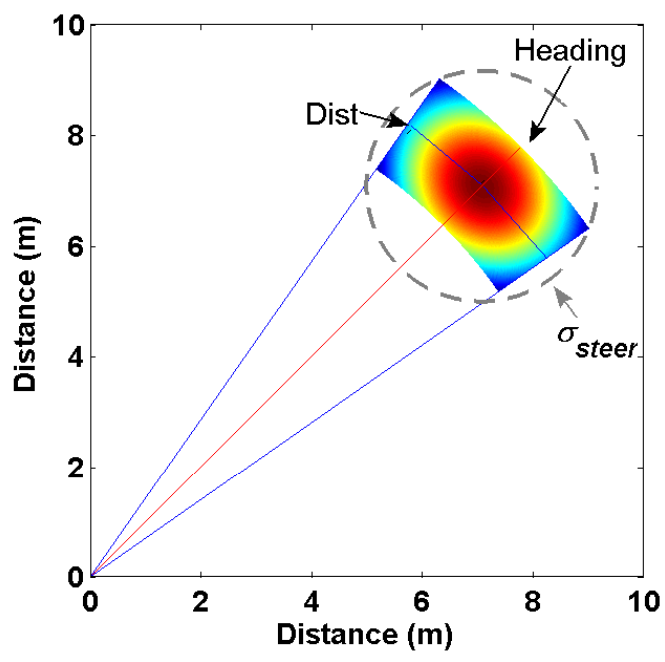

(b)

Figure 1. (a) The displacement of a UUV from $A$ to $B$ can be modeled by three components: (1) $\vec{D}+\delta \vec{D}$, where $\vec{D}$ is the estimated displacement and $\delta \vec{D}$ is the error resulted from speed estimation; (2) $(\vec{D}+\delta \vec{D}) \times \delta \vec{\theta}$, where $\delta \vec{\theta}$ is the error from steering angle estimation; (3) $\vec{\delta}_{\text {env }}$, where $\delta_{\text {env }}$ is the random shift due to the environmental currents. (b) Demonstration of DR error. The dashed circle represents the approximation using a 2-dimensional Gaussian distribution.

where $D(t)$ and $\sigma_{D}(t)$ are the distance traveled since time $t_{0}$ and the corresponding deviation, $\sigma_{\text {head }}$ is the deviation for heading error.

Assume that a UUV submerges into the water at time $t_{0}$ where it obtains an accurate GPS reading $L_{G P S}\left(t_{0}\right)$. After $t_{0}$, the location of the UUV is obtained by combining information from the initial GPS location, the dead reckoning and LBL beacon signals. In the following section, we describe our algorithmic approach to deduce the location estimates.

\subsection{Multiple Analytical Distribution Filter}

The Multiple Analytical Distribution Filter (MADF) performs probabilistic estimation of locations with dead reckoning information. As real navigation signals do not arrive at the localization device simultaneously, MADF first perform time transformation on the signal distribution to a common time point and then apply a fast intersection algorithm to deduce the result.

The time transformation of the probability distribution is based on the dead reckoning (DR). Having shown that the probability distribution of DR is not exactly symmetric, but close, we consciously neglect the asymmetry. Each DR step can be treated as normally distributed in each dimension, and can be added to the analytical distributions for any navigational instrument such as LBL beacons. For example, at time $t_{0}$ when a signal from beacon $i$ is freshly received, the probability of the range can be represented as Eq. (1). At time $t>t_{0}$, with a transformation based on DR, the beacon range would have increased variance

$$
p(x, y, z)=\frac{1}{2 \pi} \exp \left\{-\frac{\left(r^{\prime}-r_{i}\right)^{2}}{2\left(\sigma_{r}^{2}+\sigma_{D R}^{2}\right)}\right\}, \text { and } r^{\prime}=\sqrt{\left(x-x_{i}^{\prime}\right)^{2}+\left(y-y_{i}^{\prime}\right)^{2}+\left(z-z_{i}^{\prime}\right)^{2}}
$$

where $\left(x_{i}^{\prime}, y_{i}^{\prime}, z_{i}^{\prime}\right)=\left(x_{i}, y_{i}, z_{i}\right)+\left(d r_{x}, d r_{y}, d r_{z}\right)$ is the transfered location of beacon $i$, and $\left(d r_{x}, d r_{y}, d r_{z}\right)$ is the displacement estimation from dead reckoning. Here in the formula we emit the notion of $t$ to clearly represent the equation.

For UUVs the $z$ component is easily measured from pressure, and in shallow water cases the scale of $z$ is much less than $x$ or $y$. Therefore we eliminate the $z$ component in the remaining discussion and consider only two dimensions for simplicity. 


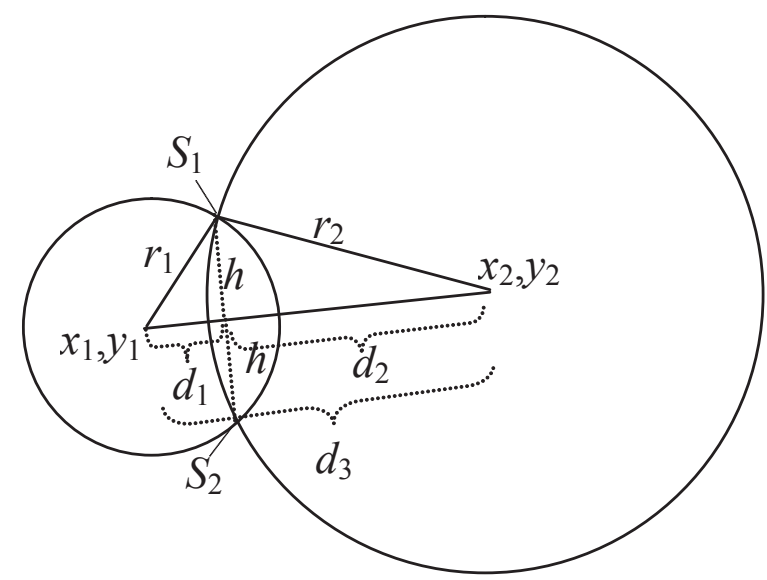

(a)

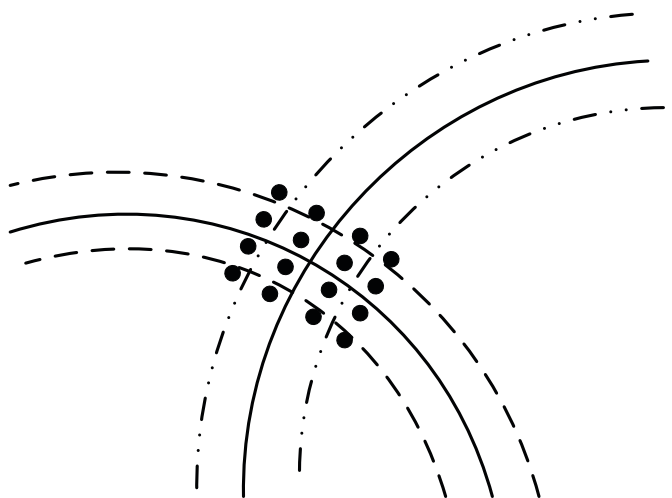

(b)

Figure 2. (a) Determining the intersection points of two annular rings. (b) Computing the intersection distribution using a small grid. Black dots are the grid points.

Once all signals are transformed to the right time instant, MADF will compute the intersections of multiple annular rings. The center points of the intersections are computed first as illustrated by a sample case of intersecting two annular rings according to Fig. 2a. The distances to two beacons, $r_{1}$ and $r_{2}$, are measured acoustically with necessary time transformation. And the location of two beacons, $\left(x_{1}, y_{1}\right)$ and $\left(x_{2}, y_{2}\right)$ are known from deployment, $d_{3}$ can be computed. Thus, $d_{1}, d_{2}$, and $h$ can be obtained by solving

$$
\left\{\begin{array}{l}
d_{1}+d_{2}=d_{3} \\
d_{1}^{2}+h^{2}=r_{1}^{2} \\
d_{2}^{2}+h^{2}=r_{2}^{2}
\end{array}\right.
$$

Thus the intersection points $S_{1}$ and $S_{2}$ can be found. Based on the rough estimation from the initial GPS reading and DR estimation, unreasonable intersection point will be eliminated and valid ones are processed to compute the probability.

Each valid intersection of two distribution fields are swept using a small grid with a fixed number of points (a sample intersection is shown in Fig. $2 \mathrm{~b})$. We denote the center location of grid $(i, j)$ as $\left(x_{i j}^{G}, y_{i j}^{G}\right)$. The boundary of the intersection area is defined as $\pm 3 \sigma$ around the center point. The probability at each grid center, $p_{i j}$, is computed based on the analytical distribution. In Section 3, we demonstrate that with a grid of only $4 \times 4$ points MADF can achieve accurate location estimation.

Once the valid intersections have been all processed, the best estimate of the location and the variance of the moment can be deduced as

$$
\begin{array}{ll}
\hat{x}=\sum_{i, j} p_{i j} x_{i j}^{G} \quad, \quad \sigma_{x}^{2}=\sum_{i, j} p_{i j}\left(x_{i j}^{G}-\hat{x}\right)^{2} ; \\
\hat{y}=\sum_{i, j} p_{i j} y_{i j}^{G} \quad, \quad \sigma_{y}^{2}=\sum_{i, j} p_{i j}\left(y_{i j}^{G}-\hat{y}\right)^{2} .
\end{array}
$$

Note that in current MADF, the estimate, $(\hat{x}, \hat{y})$, does not contribute to the next estimation. Instead the distribution from beacon readings is propagated forward or backward in time as needed.

\section{EXPERIMENTAL RESULTS}

To demonstrate the accuracy and efficiency of MADF, we built a simulation to compare the performance of MADF and particle filters with different resolutions. The advantage of a simulation is that ground truth is given. The navigation signals from beacons are constructed from the accurate distances with normally distributed noise added. This method is analogous to real acoustic systems currently available, except for the superimposed errors 
that make them unreliable in a noisy estuary with sharp salinity gradients. ${ }^{1}$ Since we are interested in comparing the computational accuracy, the environmental factors are not considered in this set of simulations.

\subsection{Particle Filter}

MADF is compared against a simple, brute force particle filter which samples a circular area around the position estimated based on the believed drift of the UUV in an environment, and with the centered on the DR position estimate. By combining the distance observation from each beacon, it then filters out the particles with low probability and assign corresponding weights on the selected particles for outputting the current estimated location. ${ }^{8-10}$ The procedure follows iterative steps:

1. Initialization: generate particles in the circle area with the center at the UUV's starting position; each particle is given an initial equal weight;

2. Using previous particles, project particles forward using the motion model;

3. Re-weight each particle by combining all beacons current range information;

4. Normalize the weight factors for all particles so that the sum of weights equals to one;

5. Output the current estimation of mobile nodes position by weighted summation of all current particles.

Given a set of range readings, an algorithm can only conclude that the likely location is at the time-shifted estimate derived from the previous location estimate using DR, and at the intersection of each pair of beacon readings. Particles must cover the entire area in order to guarantee the coverage of each likely location. Accordingly, to handle the worst case divergence of DR from beacon readings, the circular area must be kept large, sampling efficiency is low, and many particles are required. Particles are computed from scratch each time, avoiding the computational cost of resampling, but instead incur the cost of computing all the intersecting probabilities, just as MADF does.

\subsection{Accuracy Performance}

We synthesized three layout plans of beacon locations to simulate the different arrangements similar to the situation in the field. These layout plans test the accuracy of localization methods in cases with good reception of three beacons (Fig. 3a with beacons 1, 2 and 3), with limited reception of two beacons (Fig. 3a with beacons 2 and 3), and with beacon signals having small angular difference (Fig. 3b). In all cases, we use MADF and particle filters to compute the estimated location. The MADF uses a $4 \times 4$ grid to compute the probabilities around each intersection. For particle filters, we collect results from filters with 200, 500, 1000 particles since the accuracy level of particle filters is affected by the number of particles used during the computation.

Figures 4 and 5 plot the cumulative distribution of location estimation errors at $A$ and $B$ in the corresponding field. The distribution is based on 100 trials for each location. In all graphs, particle filters are labeled based on the number of particles, i.e. "PF200" is for the filter with 200 particles. Uniformly in all cases MADF has the lowest error among all filters. Among particle filters, the one with 1000 particles (PF1000) has the lowest error since it has more sampling particles to capture the signal field.

The accuracy level of all filters is affected by the field setup. For a field surrounded by three beacons, the error is much smaller for the center location $B$ (the right plot in Fig. 4a) than the corner location $A$ (the left plot in Fig. 4a). The degradation of accuracy for MADF is smaller than PF1000, which indicates that MADF is more robust to the signal quality than particle filters implemented in our simulation. In addition the accuracy of PF500 is very close to that of PF1000. Thus having extra 500 particles does not gain significant accuracy for the brute force particle filter.

When the signal reception is impaired, some beacon signal may not be heard. Figure $4 \mathrm{~b}$ plots the performance under such case. Comparing to the previous case in Fig. 4a, all filters produce higher errors. Location $B$ seems to have more degradation than location $A$ even though it is closer to the remaining two beacons. 


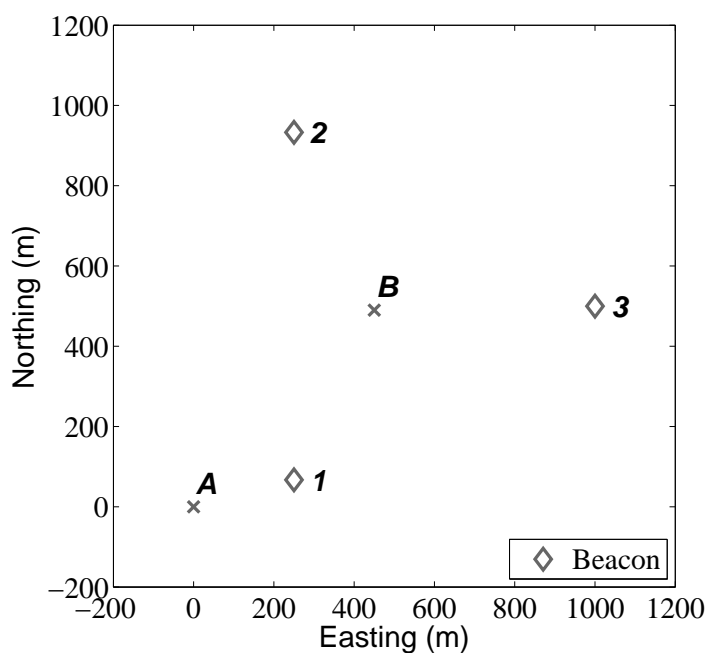

(a)

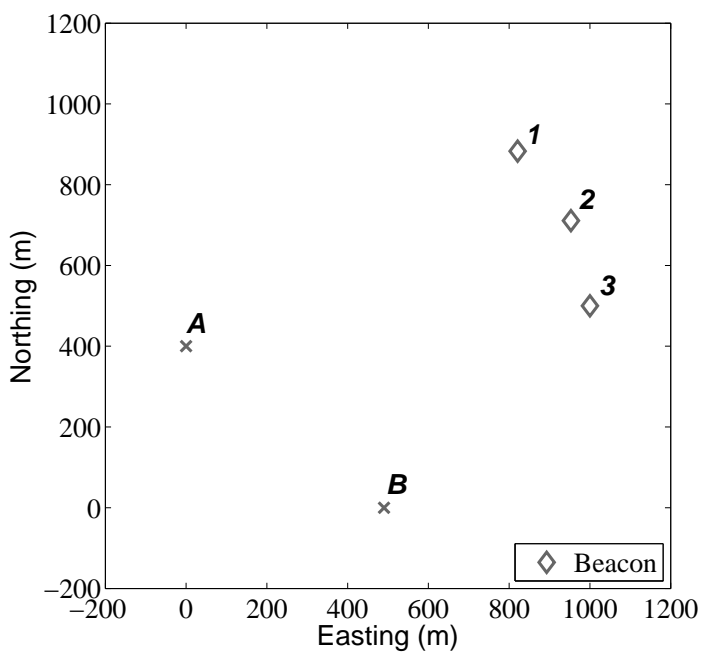

(b)

Figure 3. Simulation setup to compare MADF with particle filters. Estimation results are analyzed for locations $A$ and $B$ for each field.

During a real mission a mobile platform might travel far away from the field covered by beacons. The case in Fig. 3b simulates the far field case where the localization becomes hard even though the platform can receive signals from three beacons. As parallax error grows, the signal distribution fields are heavily overlapped, the intersection areas are very large, which presents serious challenge to all algorithms. Comparing Fig. 5 with Fig. 4 , one can see that the localization errors in the far field are almost double the previous cases. PF1000 produces similar errors with PF500 and PF250 although it uses a large amount of particles. MADF is still better than particle filters in this case. But comparing to the previous cases, the fixed-size grid is not able to cover the large intersection field, which results in much higher errors.

\subsection{Efficiency}

We compare the efficiency of the MADF algorithm to particle filters in terms of processing time. Figure 6 present the total CPU time for 100,000 iterations of each method in a field with two or three beacons. "MADF4" and "MADF8" are MADF with a $4 \times 4$ and $8 \times 8$ grid for intersection computation, respectively. The processing time of MADF is related to the number of beacons in the field, while for particle filters, the processing time depends on the number of particles since the time length is same for each particle filter in two setup of the beacon field. Clearly that MADF is using much less time than particle filters except for PF200. However PF200 has much higher errors compare to MADF4. The particle filter has to use 1000 particles to achieve smaller errors but its processing time is about 10 times larger than MADF4.

\section{CONCLUSIONS}

We propose the MADF algorithm for fast localization in field operations. It is based on the grid filter with a reduction in accuracy due to treating the DR error distribution. From our simulation, the accuracy of MADF is comparable to particle filters. MADF is computationally far more efficient in the cases where the intersection area is small, by restricting the number of probabilities computed. By contrast it is difficult to know when DR diverges from range estimates or when some range estimates contradict others. MADF is therefore a good candidate to compete with particle and grid filters in typical navigational application, such as using GPS in conjunction with an accelerometer or other means of odometry. Urban use of GPS, with signals frequently eclipsed by buildings, is an ideal application. Given access to the underlying data per satellite, GPS is exactly analogous to the LBL beacon system used in the experiments described, except that it is more accurate.

We are beginning to compare resampling particle filters against a refined version of MADF. The challenge is to keep the reliability high in the face of one or more bad readings (outside the expected range). Also, given that 

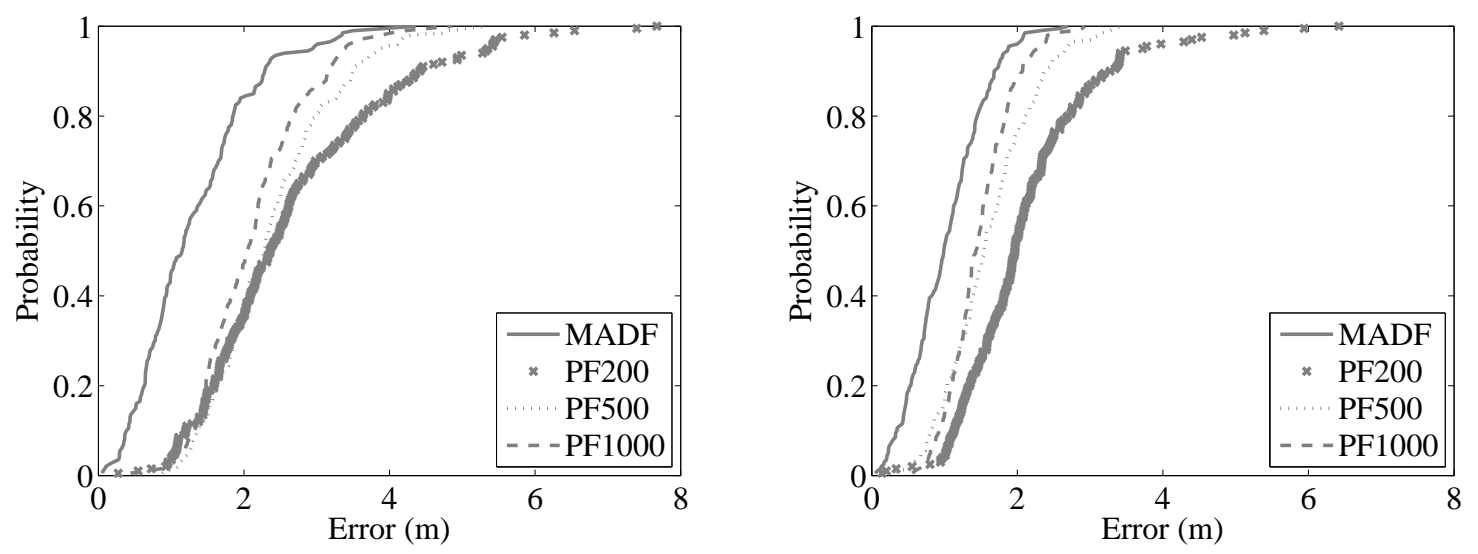

(a) With three beacons
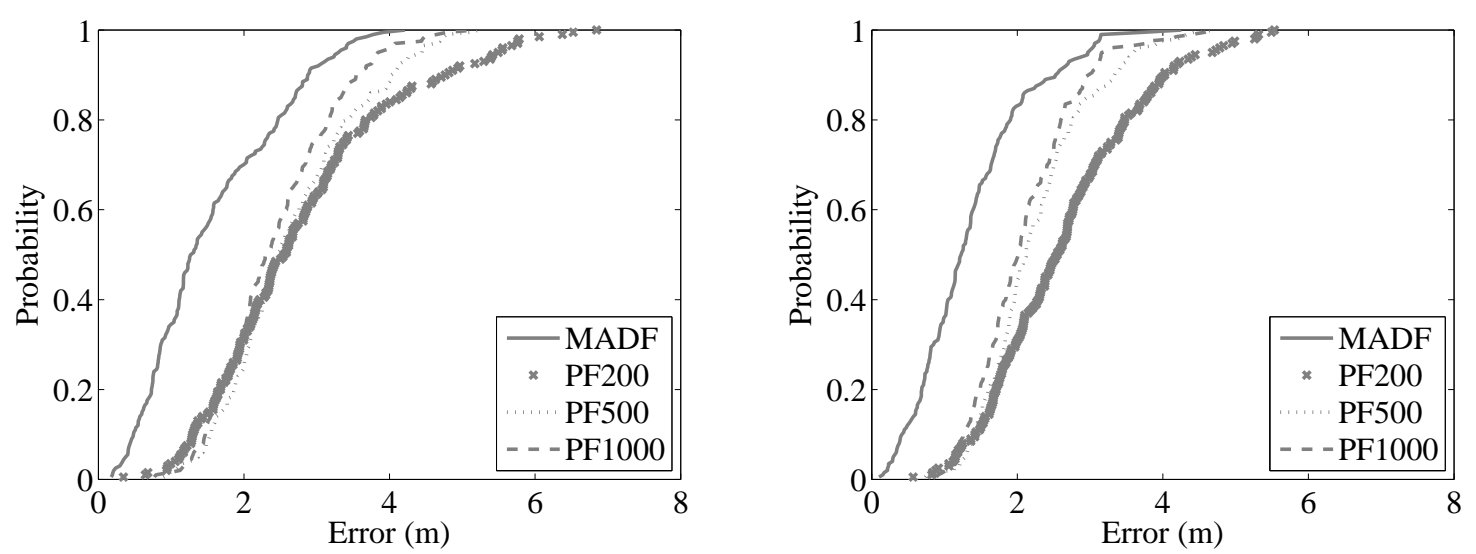

(b) With two beacons (2 and 3)

Figure 4. CDF of errors in location estimation for the field shown in Fig. 3a. The graphs on the left are for location $A$ and those on the right are for location $B$. The MADF uses a $4 \times 4$ grid to compute each intersection.

a small grid of probabilities is being computed, interpolation schemes might be used to more accurately estimate the probability distribution with fewer points. There are also other potentially useful navigational inputs with unique distributions to consider, such as RADAR/SONAR/laser range finding returning information about range and angle simultaneously, and using bottom scanning SONAR to recognize local features as a very precise way to measure relative motion.

\section{ACKNOWLEDGMENTS}

This research was partially supported by the Office of Naval Research, Grant \#N00014-05-0632.

\section{REFERENCES}

[1] Shi, H., Kruger, D., and Nickerson, J. V., "Incorporating environmental information into underwater acoustic sensor coverage estimation in estuaries," in [Proceedings of the AFCEA/IEEE Military Communications Conference (MILCOM)], (October 2007).

[2] Kantor, G. and Singh, S., "Preliminary results in range-only localization and mapping," in [Proceedings of IEEE International Conference on Robotics and Automation (ICRA)], 1818-1823 vol.2 (2002).

[3] Hu, L. and Evans, D., "Localization for mobile sensor networks," in [Proceedings of the International Conference on Mobile Computing and Networking (MobiCom)], 45-57 (2004). 

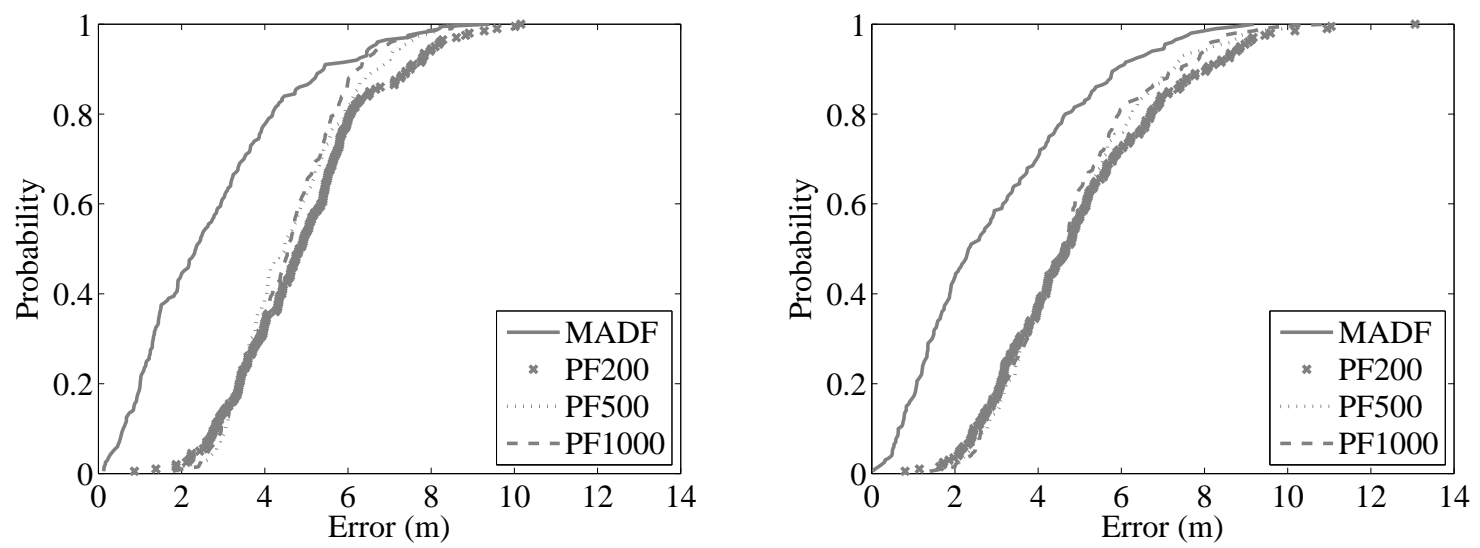

Figure 5. CDF of errors in location estimation for the field shown in Fig. 3b. The left graph is for location $A$ and the right one is for location $B$. The MADF uses a $4 \times 4$ grid to compute each intersection.

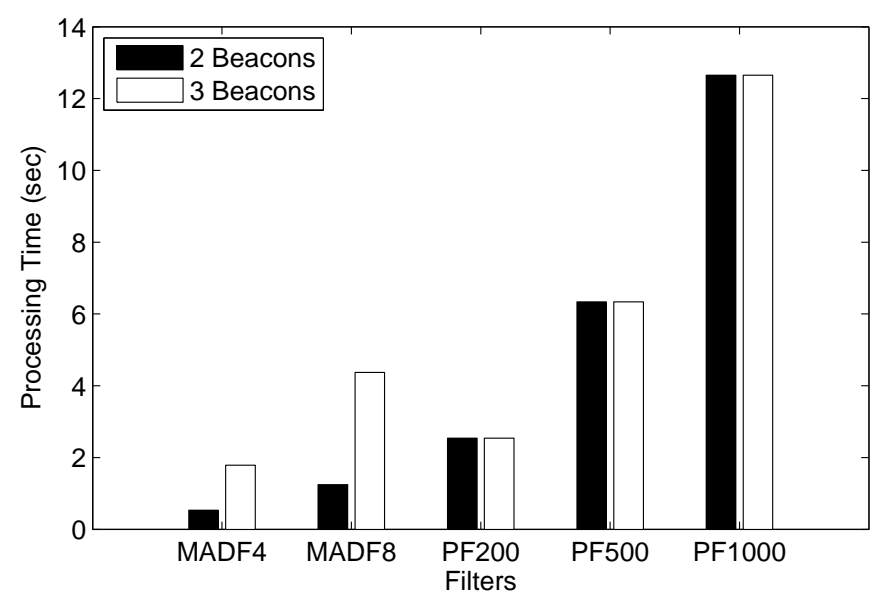

Figure 6. Processing time for 100,000 iterations of MADF and particle filters.

[4] Welch, G. and Bishop, G., "An introduction to the Kalman Filter," Technical Report 95-041, University of North Carolina at Chapel Hill, Dept. of Computer Science.

[5] Chen, Y., Francisco, J.-A., Trappe, W., and Martin, R., "A practical approach to landmark deployment for indoor localization," in [Proceedings of the Third Annual IEEE Communications Society Conference on Sensor and Ad Hoc Communications and Networks (SECON)], 1, 365-373 (Sept. 2006).

[6] Yang, J., Chen, Y., Lawrence, V., and Swaminathan, V., "Robust wireless localization to attacks on access points," in [Proceedings of IEEE Sarnoff Symposium], 1-5 (30 2009-April 1 2009).

[7] Thrun, S., Burgard, W., and Fox, D., "A probabilistic approach to concurrent mapping and localization for mobile robots," Machine Learning 31(1-3), 29-53 (1998).

[8] Baggio, A. and Langendoen, K., "Monte Carlo localization for mobile wireless sensor networks," in [Proceedings of Second International Conference on Mobile Ad-hoc and Sensor Networks (MSN 2006)], 317-328 (2006).

[9] Doucet, A., de Freitas, J. F. G., and Gordon, N. J., eds., [Sequential Monte Carlo Methods in Practice], Springer-Verlag (2001).

[10] Wang, W. and Zhu, Q., "RSS-based Monte Carlo localisation for mobile sensor networks," IET Communications 2, 673-681 (May 2008).

[11] Kruger, D., Shi, H., Samaan, M., Chesley, D., Nickerson, J. V., and Imas, L., "High speed localization using a multiple analytical distribution filter," in press with Journal of Underwater Acoustics (U.S. Navy) . 